\title{
Isolation of Comamonas Species from the Stool Samples of Patients with Underlying Gastrointestinal Pathology
}

\author{
Rachana Kannambath ${ }^{1}$, Pheba Stanley ${ }^{2}$, Pazhanivel Mohan ${ }^{3}$, Jharna Mandal ${ }^{4}$
}

\begin{abstract}
Comamonas species are saprophytes, thought to be rarely associated with clinical infections. We report five cases of Comamonas species associated with gastrointestinal $(\mathrm{Gl})$ manifestations isolated over a period of 2 years. All these patients had underlying $\mathrm{Gl}$ pathologies like malignancy or inflammatory bowel disease. This report tends to introspect on the clinical significance of such rare pathogens in stool samples and emphasize its possible role in causing $\mathrm{Gl}$ infections.

Keywords: Comamonas aquatica, Comamonas testosteroni, Gastrointestinal infection.

Journal of Gastrointestinal Infections (2021): 10.5005/jp-journals-10068-3049
\end{abstract}

\section{INTRODUCTION}

Comamonas are aerobic, oxidase-positive gram-negative bacilli, which belongs to the family Comamonadaceae in the Pseudomonas rRNA homology group III. ${ }^{1}$ They emerged as a separate genus in 1985 with a single species C. terrigena. Later in 1987, two more species, $P$. testosteroni and $P$. acidovorans were reclassified into this genus. C. acidovorans were later reclassified as Delftia acidovorans. ${ }^{1}$ C. terrigena initially comprised of three DNA hybridization groups which was later described as three distinct species, namely C. terrigena, C. aquatica, and C. kerstersii. ${ }^{2}$ Comamonas spp. are generally present in natural habitats such as soil, water, and plants. They were considered non-pathogenic saprophytes until 1987, after which a few case reports emerged, where Comamonas spp. were isolated from various clinical samples. Most commonly, they were associated with intra-abdominal infections. The most common species reported are C. testosteroni followed by C. kerstersii. ${ }^{3}$ Here, we describe five cases of isolation of Comamonas spp. (four of C. aquatica and one of C. testosteroni) from stool samples of patients with gastrointestinal (Gl) manifestations over a period of 2 years (January 2019-December 2020).

\section{Case Descriptions}

\section{Case 1}

A 60-year-old man presented with complaints of passage of watery stool along with hematochezia. Stool frequency was 5-7 times per day and associated with crampy abdominal pain. There was a history of significant weight loss over the past 4 months. On per abdominal examination, a mass of approximately $5 \times 4 \mathrm{~cm}$ was felt in the left iliac fossa. On examination, vitals and other systems were within normal limits. His routine blood investigation showed mild anemia and a slight elevation of total leukocyte count. A stool sample was sent for microscopy and culture to rule out any Gl infection. There were no pus cells, red blood cells (RBCs), or parasitic elements on microscopy. Stool culture was done on two selective media, MacConkey agar, and Xylose Lysine Deoxycholate agar (XLD) and an enrichment media-Selenite $F$, which was further subcultured onto the above mentioned selective media after 16-18 hours. On direct plating, pale non-lactose-fermenting colonies were noted on the MacConkey agar (Fig. 1), which were oxidase-positive and

\begin{abstract}
${ }^{1-4}$ Department of Microbiology, Jawaharlal Institute of Postgraduate Medical Education and Research, Puducherry, India

Corresponding Author: Jharna Mandal, Department of Microbiology, Jawaharlal Institute of Postgraduate Medical Education and Research, Puducherry, India, e-mail: drjharna@gmail.com

How to cite this article: Kannambath $R$, Stanley $P$, Mohan $P$, et al. Isolation of Comamonas Species from the Stool Samples of Patients with Underlying Gastrointestinal Pathology. J Gastrointest Infect 2021;11:47-50.
\end{abstract}

Source of support: Nil

Conflict of interest: None

red-colored colonies on XLD. The colonies were identified using conventional biochemical tests and automated method MALDI TOF MS (VITEK MS V3/KB V3.2.0, bioMerieux, Marcy L'Etoile, France). The biochemical reactions were as follows. Indole was not produced, citrate was not utilized, urea was not hydrolyzed, Kligler iron agar showed alkaline slant and alkaline butt without gas or hydrogen

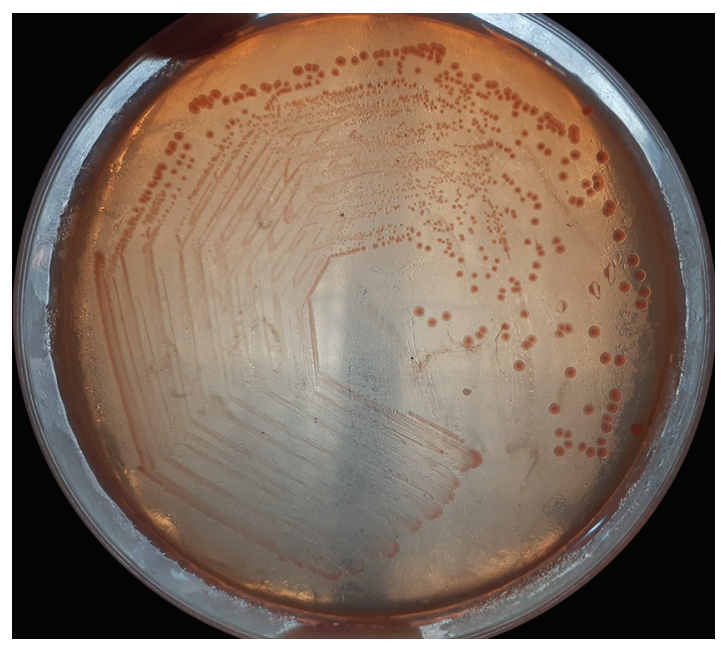

Fig. 1: Pale non-lactose-fermenting colonies of Comamonas spp. on MacConkey agar

() The Author(s). 2021 Open Access This article is distributed under the terms of the Creative Commons Attribution 4.0International License (https://creativecommons. org/licenses/by-nc/4.0/), which permits unrestricted use, distribution, and non-commercial reproduction in any medium, provided you give appropriate credit to the original author(s) and the source, provide a link to the Creative Commons license, and indicate if changes were made. The Creative Commons Public Domain Dedication waiver (http://creativecommons.org/publicdomain/zero/1.0/) applies to the data made available in this article, unless otherwise stated. 
sulfide. MALDI TOF MS identified the colonies as C. aquatica with a 99.9\% confidence level.

Antimicrobial susceptibility testing was performed as per the CLSI 2020 using Pseudomonas aeruginosa as the reference standard. It was found susceptible to ampicillin, cotrimoxazole, ceftriaxone, piperacillin-tazobactam, cefoperazone sulbactam, and meropenem and resistant to ciprofloxacin. The patient was treated with ampicillin for 5 days. Ultrasound of abdomen and pelvis revealed cholelithiasis and bilateral medical renal disease. Sigmoidoscopy of the patient showed ulceroproliferative growth at the rectosigmoid junction. CECT of the abdomen revealed a circumferential wall thickening in the mid sigmoid colon with a serosal breach involving the posterior wall. Biopsy from the growth was suggestive of villous adenoma with low-grade dysplasia. The patient underwent roboticassisted anterior resection, intraoperative, and postoperative period was uneventful. The patient was discharged in stable condition, tolerating a normal diet and advised for follow-up.

\section{Case 2}

A 62-year-old man with 10 years history of diabetes being managed on oral hypoglycemic agents presented with complaints of vomiting, loose stool, abdominal distension, and intolerance for solid and semisolid food for the past 3 weeks. He also noted the passage of dark colored stool for the past 3 weeks. On examination, the abdomen was soft, nontender, with no palpable mass or organomegaly and shifting dullness was present. Vitals and examination of other systems were within normal limits. His blood investigations revealed a low hemoglobin count of $6.8 \mathrm{~g} / \mathrm{dL}$, with a slightly elevated total leukocyte count of $16,080 \mathrm{~mm}^{3}$ and decreased albumin level of $2.4 \mathrm{~g} / \mathrm{dL}$. Ultrasound of abdomen revealed massive ascites with septations and omental thickening with a few nodules. In upper Gl endoscopy, circumferential growth in the gastroesophageal junction extending to the body of the stomach was noted. Multiple biopsies were taken, which revealed the evidence of moderately differentiated adenocarcinoma of the gastroesophageal junction. Ascitic fluid cytology was also positive for malignant cells. A stool sample was sent for microscopy and culture. There were no pus cells, RBCs, or parasitic elements on microscopy. Stool culture and identification was performed as described in case 1, and the primary culture yielded C.aquatica. The isolate was susceptible to ampicillin, ceftriaxone, ciprofloxacin, cotrimoxazole, cefoperazone sulbactam, and meropenem. The patient was given injection ciprofloxacin 200 mg IV 12 hourly and injection metronidazole 500 mg IV 8 hourly. He was planned for best supportive care and chemotherapy with oral capecitabine and follow-up in a regional cancer center. At the time of discharge, the patient was stable, tolerating oral feeds, and vomiting subsided.

\section{Case 3}

A 43-year-old woman, known case of ulcerative colitis on irregular medications, came with complaints of increased stool frequency, 8-10 episodes per day, associated with blood in stool, urgency, tenesmus, and pain abdomen for the past 1 month. On examination, vitals and all systems were within normal limits. Routine blood investigations showed mild anemia (Hb: $9.8 \mathrm{~g} / \mathrm{dL}$, MCH: 24.9 pg, MCHC: $30 \mathrm{~g} / \mathrm{dL}$ ) and elevated ESR (110 mm/hour). Stool culture was sent to rule out any associated infection. Pus cells and RBCs were present in microscopy. Culture yielded C. aquatica susceptible to ampicillin, ceftriaxone, cotrimoxazole, cefoperazone sulbactam, meropenem, and resistant to ciprofloxacin. The patient was treated with oral cotrimoxazole for 3 days. Colonoscopy findings were suggestive of severe flare of ulcerative colitis. Therefore, the patient was initially started on tablet mesalamine and mesalamine enema, which was subsequently changed to sulfasalazine due to evidence of acute sacroiliitis. The patient improved symptomatically and was discharged in stable condition.

\section{Case 4}

A 65-year-old man presented with complaints of fecal incontinence and pain during defecation for the past 8 months. On general examination, bilateral inguinal lymph nodes were palpable and vitals were stable. A circumferential growth of $4 \times 4 \mathrm{~cm}$, firm in consistency was felt on per rectal examination, which did not bleed on touch. Other systems examinations were within normal limits. A stool sample was sent for microscopy and culture to rule out any infection. Pus cells and RBCs were present on microscopy, and C. testosteroni was isolated from the primary stool culture, which was susceptible to ampicillin, ceftriaxone, cotrimoxazole, cefoperazone, sulbactam, and meropenem but resistant to ciprofloxacin. The patient was treated with injection ampicillin for 5 days. MRI scan of pelvis and screening of upper abdomen revealed a mass involving the proximal two-third portion of the rectum, rectosigmoid junction and distal sigmoid colon along with florid regional lymph nodes and multiple discrete regional lymph nodes on either side. A solitary gallstone was also detected. Biopsy findings were suggestive of well-differentiated adenocarcinoma of the rectum. A wholebody FDG-PET scan revealed mesorectal lymph node metastasis. The patient was managed with neoadjuvant chemotherapy and radiotherapy.

\section{Case 5}

A 62-year-old man presented with complaints of passing loose stool for the past 2 months, 6-7 episodes per day, occasionally associated with blood in the stool. On examination, vitals, all systems, and baseline laboratory parameters were within normal limits. A provisional diagnosis of inflammatory bowel disease was considered. On chest X-ray, there was bilateral upper lobe consolidation, CECT of the thorax revealed bilateral apical lobe cavities. The patient was worked up to diagnose pulmonary tuberculosis/ileocecal tuberculosis. The sputum acid-fast smear and the cartridge-based nucleic acid amplification test for Mycobacterium tuberculosis turned out to be negative. A stool sample was sent for microscopy and culture. Pus cells were present on microscopy, and C. aquatica was isolated from the primary stool culture susceptible to ampicillin, ceftriaxone, cefoperazonesulbactam, and meropenem but resistant to ciprofloxacin and cotrimoxazole. The patient was treated with ampicillin for 5 days, following which diarrhea subsided. The patient is under further evaluation for his respiratory pathology.

The clinical details and laboratory findings of all the patients have been summarized in Table 1.

\section{Discussion}

Comamonas spp. are nonfermenting bacteria, rarely associated with clinical infections. The majority of the cases reported till now includes intra-abdominal infections and sepsis. ${ }^{4}$ Most of these had been associated with predisposing appendicular perforation. ${ }^{3}$ There are a few reports which describe the isolation of Comamonas spp. from the stool sample. C. testosteroni was isolated from the stool sample of an elderly with a history of colostomy and presented with watery diarrhea. ${ }^{5}$ Biswas et al. reported the isolation of C. kerstersii 
Table 1: Clinical and laboratory findings of the cases

\begin{tabular}{|c|c|c|c|c|c|c|c|c|c|c|c|c|}
\hline \multirow[b]{2}{*}{ Cases } & \multirow[b]{2}{*}{ Age/gender } & \multirow[b]{2}{*}{ Clinical features } & \multirow[b]{2}{*}{ Coexisting conditions } & \multirow[b]{2}{*}{ Stool microscopy } & \multirow{2}{*}{$\begin{array}{c}\text { Organism isolated } \\
\text { in culture }\end{array}$} & \multicolumn{7}{|c|}{ Antimicrobial susceptibility } \\
\hline & & & & & & $A$ & $\mathrm{Ci}$ & Cfs & Pt & $M$ & Cot & $C f$ \\
\hline 1 & 60/Male & $\begin{array}{l}\text { Loose stool, } \\
\text { hematochezia }\end{array}$ & $\begin{array}{l}\text { Villous adenoma of } \\
\text { rectum, gallstones }\end{array}$ & $\begin{array}{l}\text { No pus cells, RBC's } \\
\text { or parasitic elements }\end{array}$ & C. aquatica & $\mathrm{S}$ & $S$ & $S$ & $S$ & $\mathrm{~S}$ & $S$ & $\mathrm{R}$ \\
\hline 2 & 65/Male & $\begin{array}{l}\text { Vomiting, loose stool, } \\
\text { abdominal distension }\end{array}$ & $\begin{array}{l}\text { Adenocarcinoma } \\
\text { stomach, Diabetes } \\
\text { mellitus }\end{array}$ & $\begin{array}{l}\text { No pus cells, RBC's } \\
\text { or parasitic elements }\end{array}$ & C. aquatica & $\mathrm{S}$ & $\mathrm{S}$ & $S$ & $S$ & $S$ & $S$ & $\mathrm{~S}$ \\
\hline 3 & 43/Female & $\begin{array}{l}\text { Increased } \\
\text { frequency of } \\
\text { stool, blood in stool, } \\
\text { tenesmus and pain } \\
\text { abdomen }\end{array}$ & Ulcerative colitis & $\begin{array}{l}\text { Pus cells and RBC's } \\
\text { present, no } \\
\text { parasiticelements }\end{array}$ & C. aquatica & $\mathrm{S}$ & $\mathrm{S}$ & $S$ & $S$ & $\mathrm{~S}$ & $S$ & $\mathrm{R}$ \\
\hline 4 & 66/Male & $\begin{array}{l}\text { Fecal } \\
\text { incontinence, pain } \\
\text { while defecation }\end{array}$ & $\begin{array}{l}\text { Adenocarcinoma } \\
\text { rectum, gallstones }\end{array}$ & $\begin{array}{l}\text { Pus cells and RBC's } \\
\text { present, no parasitic } \\
\text { elements }\end{array}$ & C. testosteroni & $\mathrm{S}$ & $\mathrm{S}$ & $\mathrm{S}$ & $S$ & $\mathrm{~S}$ & $S$ & $\mathrm{R}$ \\
\hline 5 & 62/Male & $\begin{array}{l}\text { Loose stool, } \\
\text { occasionally associated } \\
\text { with blood }\end{array}$ & Ileocaecal tuberculosis & $\begin{array}{l}\text { Pus cells present, no } \\
\text { parasitic elements }\end{array}$ & C. aquatica & $\mathrm{S}$ & $\mathrm{S}$ & $\mathrm{S}$ & $S$ & $\mathrm{~S}$ & $\mathrm{R}$ & $\mathrm{R}$ \\
\hline
\end{tabular}

A, ampicillin; Ci, ceftriaxone; Cfs, cefoperazone-sulbactam; Pt, piperacillin tazobactam; M, meropenem; Cot, cotrimoxazole; Cf, ciprofloxacin; S, susceptible; R, resistant

from 27 stool samples from patients who presented with diarrhea over a period of 2 years. There are several reports of isolation of C. testosteroni from cases of bacteremia, septic shock, appendicitis, catheter-related infections, infective endocarditis, etc. ${ }^{6,7}$

The reports of isolation of $C$. aqautica from clinical samples are very scanty. After a thorough literature search, no other account of the isolation of $C$. aquatica from the stool sample was found. Interestingly, four out of five of our isolates were identified as C. aquatica. There are a few reports of sepsis caused by Comamonas spp. where Gl colonization due to environmental exposure to contaminated water was thought to be the probable source. ${ }^{1,8,9}$ C. testosteroni was isolated from the blood of a 12-month-old child who presented with acute gastroenteritis, which later progressed into sepsis. ${ }^{10} \mathrm{C}$. kerstersii was isolated along with Bacteroides fragilis from the blood sample of an elderly patient with sudden onset of fever, diarrhea, and vomiting, which later progressed to sepsis. An abdominal computed tomography scan revealed diverticulosis, and the patient also had a history of drinking water from the river. All these factors prompted the authors to think of gut as the source of infectiont. ${ }^{8} \mathrm{C}$. aquatica has been reported from the blood sample of a patient with septic shock, whose initial presentation was with diarrhea and fever. The patient was a known diabetic and had a history of ischemic heart disease and removal of the sigmoid polyp. ${ }^{4}$ Although the organism was not isolated from the stool, the potential of this organism to translocate from the gut to cause systemic infection has to be considered, owing to the initial presentation with diarrhea and the coexisting conditions of the patient. ${ }^{4}$ They are also known to be colonizing hospital devices such as intravenous lines, respiratory equipment, and humidifiers. But most commonly, they are associated with community-acquired than nosocomial infections. ${ }^{1}$

Although they are considered to be low-virulent organisms, pan-genome and core genome analysis of various species of Comamonas have revealed that they possess diverse virulence mechanisms such as factors for adherence, antiphagocytosis factors, motility systems, and metabolic enzymes for adaptation. ${ }^{11}$ They possess several advanced environmental sensors and signaling systems, which enables them to persist in the environment and form biofilms. ${ }^{11}$ All the five patients in our cases were from rural areas, and no specific history of environmental exposure to contaminated water could be elicited. Most of the previous cases reported were associated with some other comorbidity or immunosuppression such as malignancy, chronic liver disease, HIV, diabetes mellitus, etc. Three of the patients in our case were associated with GI malignancy in which one of them also had diabetes, and one had inflammatory bowel disease. Since all had underlying GI pathology, it is difficult to determine whether Comamonas spp. were responsible for the clinical symptoms or were primarily due to the underlying pathology. Nevertheless, Comamonas spp. could be contributing to any of these clinical features, which needs further exploration. But as it was isolated as the predominant pathogen compared to the normal gut microbial flora in all the five cases, and considering the previous reports of association with disease, one cannot ignore its presence. Two of our patients had ultrasonographic evidence of gallstones. A similar observation was noted in another study, where they considered gallbladder to be a potential source of harboring this organism since Comamonas spp. have the genes for the utilization of aromatic and short-chain fatty acids as the source of carbon. ${ }^{5}$

In many of the previous reports, Comamonas spp. were found to be susceptible to aminoglycosides, cephalosporins, cotrimoxazole, ciprofloxacin, etc., and the majority recovered with appropriate antibiotic therapy ${ }^{6,7}$ In our case, except for one isolate of C. aquatica, all others were found to be resistant to ciprofloxacin. Some workers have reported similar observations. ${ }^{4,5,9}$ But whether antimicrobial agents are required for the treatment is obscure.

\section{Conclusion}

Inadequate phenotypic methods for the identification of Comamonas spp. could be a reason for its underreporting. There is a chance that Comamonas spp. could be misidentified as Pseudomonas spp. using the routine, conventional biochemical reactions. Although the advent of automated identification systems has increased the detection rate, species-level identification is still a challenge as many of the automated systems had a limited 
number of Comamonas spp. in their databases. ${ }^{12}$ There is a high chance of ignoring such pathogens as commensals, especially from stool samples.

\section{References}

1. Almuzara $M$, Barberis $C$, Veiga $F$, et al. Unusual presentations of Comamonas kerstersii infection. New Microbes New Infect 2017;19: 91-95. DOI: 10.1016/j.nmni.2017.07.003.

2. Wauters G, De Baere T, Willems A, et al. Description of Comamonas aquatica comb. nov. and Comamonas kerstersii sp. nov. for two subgroups of Comamonas terrigena and emended description of Comamonas terrigena. Int J Syst Evol Microbiol 2003;53(Pt 3): 859-862. DOI: 10.1099/ijs.0.02450-0.

3. Almuzara MN, Cittadini R, Ocampo CV, et al. Intra-abdominal infections due to Comamonas kerstersii. J Clin Microbiol 2013;51(6): 1998-2000. DOI: 10.1128/JCM.00659-13.

4. Kaeuffer C, Schramm F, Meyer A, et al. First case of Comamonas aquatica bacteremia complicated by septic shock. Med Mal Infect 2018;48(8):540-542. DOI: 10.1016/j.medmal.2018.08.004.

5. Farooq S, Farooq R, Nahvi N. Comamonas testosteroni: is it still a rare human pathogen? Case Rep Gastroenterol 2017;11(1):42-47. DOI: 10.1159/000452197.
6. Tiwari S, Nanda M. Bacteremia caused by Comamonas testosteroni an unusual pathogen. J Lab Physicians 2019;11(1):87-90. DOI: 10.4103/ JLP.JLP_116_18.

7. Farshad S, Norouzi F, Aminshahidi M, et al. Two cases of bacteremia due to an unusual pathogen, Comamonas testosteroni in Iran and a review literature. J Infect Dev Ctries 2012;6(6):521-525. DOI: 10.3855/ jidc. 2215

8. Opota O, Ney B, Zanetti G, et al. Bacteremia caused by Comamonas kerstersii in a patient with diverticulosis. J Clin Microbiol 2014;52(3):1009-1012. DOI: 10.1128/JCM.02942-13.

9. Biswas JS, Fitchett J, O'Hara G. Comamonas kerstersii and the perforated appendix. J Clin Microbiol 2014;52(8):3134. DOI: 10.1128/ JCM.00909-14.

10. Ruziaki WA, Hashami HA. Unusual pathogen Comamonas testosterone sepsis following gastroenteritisin a 12 months old child: case report and literature review. Am J Med Case Rep 2017;5:148-150. DOI: 10.12691/ ajmcr-5-6-4.

11. WuY,Zaiden N, Cao B. The core-and pan-genomic analyses of the genus Comamonas: from environmental adaptation to potential virulence. Front. Microbiol 2018;9:3096. DOI: 10.3389/fmicb.2018.03096.

12. Zhou YH, Ma HX, Dong ZY, et al. Comamonas kerstersii bacteremia in a patient with acute perforated appendicitis: a rare case report. Medicine 2018;97(13):e9296. DOI: 10.1097/MD.0000000000009296. 